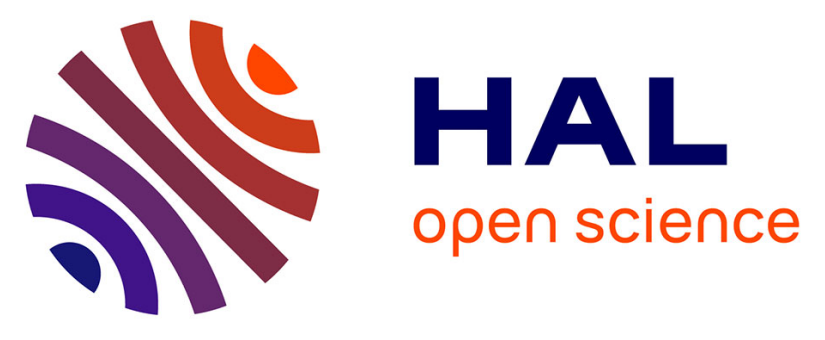

\title{
Engineered toxin-intein antimicrobials can selectively target and kill antibiotic-resistant bacteria in mixed populations
}

\author{
Rocío López-Igual, Joaquin Bernal-Bayard, Alfonso Rodríguez-Patón, \\ Jean-Marc Ghigo, Didier Mazel
}

\section{To cite this version:}

Rocío López-Igual, Joaquin Bernal-Bayard, Alfonso Rodríguez-Patón, Jean-Marc Ghigo, Didier Mazel. Engineered toxin-intein antimicrobials can selectively target and kill antibiotic-resistant bacteria in mixed populations. Nature Biotechnology, 2019, 37 (7), pp.755-760. 10.1038/s41587-019-0105-3 . pasteur-02558499

\section{HAL Id: pasteur-02558499}

https://hal-pasteur.archives-ouvertes.fr/pasteur-02558499

Submitted on 29 Apr 2020

HAL is a multi-disciplinary open access archive for the deposit and dissemination of scientific research documents, whether they are published or not. The documents may come from teaching and research institutions in France or abroad, or from public or private research centers.
L'archive ouverte pluridisciplinaire HAL, est destinée au dépôt et à la diffusion de documents scientifiques de niveau recherche, publiés ou non, émanant des établissements d'enseignement et de recherche français ou étrangers, des laboratoires publics ou privés. 


\section{Engineered toxin-intein antimicrobials can selectively target and}

2 kill antibiotic-resistant bacteria in mixed populations

3

4

5

6 Rocío López-Igual ${ }^{1 a}$, Joaquín Bernal-Bayard², Alfonso Rodríguez-Patón ${ }^{3}$, Jean-Marc Ghigo ${ }^{2}$

7 and Didier Mazel ${ }^{1 *}$.

$8{ }^{1}$ Unité de Plasticité du Génome Bactérie, Département Génomes et Génétique, Institut

9 Pasteur, UMR3525, CNRS, Paris, France.

10 'Unité de Génétique des Biofilms, Département Microbiologie, Institut Pasteur, Paris,

11 France.

12 Unniversidad Politécnica de Madrid, Departamento de Inteligencia Artificial, ETSIINF, 28040

13 Madrid, Spain.

14

15

18 a Current Adress: Instituto de Bioquímica Vegetal y Fotosíntesis, CSIC and Universidad de

19 Sevilla, Seville, Spain.

$20{ }^{*}$ Correspondence should be addressed to D.M. (mazel@pasteur.fr) 
21 Targeted killing of pathogenic bacteria without harming beneficial members of host

22 microbiota holds promise as a strategy to cure disease, and limit both antimicrobial-

23 related dysbiosis and development of antimicrobial resistance. We engineer toxins

24 that are split by inteins and deliver them by conjugation into a mixed population of

25 bacteria. Our toxin-intein antimicrobial is only activated in bacteria that harbor

26 specific transcription factors. We apply our antimicrobial to specifically target and kill

27 antibiotic resistant Vibrio cholerae in complex populations gathering various bacterial

28 species. We found that $100 \%$ of antibiotic resistant $V$. cholerae receiving the plasmid

29 were killed. Escape mutants were extremely rare (10-6-10-8). We demonstrate that

30 conjugation and specific killing of targeted bacteria is functional in the microbiota of

31 zebrafish and crustacean larvae, which are natural hosts for Vibrio spp. Toxins split

32 with inteins could form the basis of a range of precision antimicrobials which would

33 kill both Gram - and Gram + pathogens. With the advent of the antibiotic era, infectious diseases were thought to be under control, but worldwide emergence of antibiotic-resistant bacteria has occurred, owing to the 
39 bacteria could be the main cause of death by $2050^{1}$ unless new classes of antimicrobials are

40 developed.

41 Broad spectrum antimicrobials indiscriminately kill bacteria which can result in

42 microbiota dysbiosis and concomitant health sequelae. Moreover, antibiotic that have non-

43 specific targets can select for antibiotic resistance, which is mainly acquired by horizontal

44 gene transfer among bacteria in communities ${ }^{2}$. Alternatives to broad spectrum antibiotics

45 include bacteriocins, which kill a subset of bacterial species or strains, and will not provoke a

46 superinfection ${ }^{3}$. Other targeted antimicrobials have also been reported, including CRISPR-

47 Cas antimicrobials ${ }^{4-6}$, phage therapy ${ }^{7}$ and local release of toxins ${ }^{8}$.

48 We set out to design antimicrobials to specifically kill antibiotic-resistant Vibrio

49 cholerae. To mediate bacterial killing we chose the toxin component of type II bacterial toxin-

50 antitoxin (TA) systems, which are involved in stabilization of plasmids, prophages and

51 superintegrons $^{9}$. Type II toxin and antitoxins are proteins ${ }^{9}$. The toxin targets conserved

52 bacterial cellular functions which reduces the potential for development of resistance. Each

53 antitoxin is highly specific for the cognate toxin, and nonspecific toxin-antitoxin interactions

54 are counterselected ${ }^{10}$. Our antimicrobial design relies on the regulation of type II TA

55 transcription by highly specific transcription factors (TF). This means that activation of the

56 toxin, and concomitant killing, of individual members of mixed bacterial populations is

57 feasible if a targeted bacterial species expresses the Type II toxin-regulating transcription 
58 factor. We validated our approach by showing that we could selectively kill antibiotic-

59 resistant $V$. cholerae present in mixed populations.

60 V. cholerae causes between 21000 and 143000 deaths from cholera per year ${ }^{11}$.

61 The most recent cholera pandemics involved the $\mathrm{O} 1$ and $\mathrm{O} 139$ serogroups. Virulence in $V$.

62 cholerae is coordinated by the master transcriptional activator ToxR, which regulates the

63 ToxR regulon ${ }^{12}$, which includes the cholera toxin genes. Cholera epidemics are associated

64 with antibiotic resistance owing to resistance genes present on an integrative and

65 conjugative element (ICE) named SXT (from sulfamethoxazole and trimethoprim resistance).

66 SXT can carry genes that confer resistance to sulfamethoxazole (sul2), trimethoprim (dfrA1

67 and $d f r 18)$, streptomycin ( $\operatorname{str} B)$, chloramphenicol (floR) and tetracycline (tetA) and was first

68 described in $V$. cholerae serogroup $0139^{13}$. SXT also encodes functions promoting its

69 excision, dissemination by conjugation, and integration, as well as the transcription factors

70 that control expression of these functions ${ }^{13}$.

71 Our previous experience with type II toxins ${ }^{14,15}$ taught us that basal expression of a

72 full-length toxin gene from $\mathrm{P}_{\mathrm{BAD}}$ is sufficient to kill the E. coli host. To avoid this, we

73 designed a genetic module containing a toxin split by an intein, and in our module the split

74 toxin-intein can only be activated by ToxR. Inteins are protein sequences embedded into a

75 host protein (extein) from which they are autocatalytically excised in a process called protein

76 splicing. During protein splicing the intein ligates the extein extremities and allows the 
77 reconstitution of the mature protein. In nature, a few examples of split inteins also exist

78 allowing the assembly of a single protein from two genes ${ }^{16}$. We split the type II toxin gene

$79 \operatorname{ccdB}$ (Plasmid pToxInt, Supplementary Fig. 1) into two parts, each of which is associated

80 with half of a split intein. Split inteins have been used in several biotechnological tools ${ }^{17}$ and

81 enable control of toxic protein functions in vivo ${ }^{18}$. We used the split intein DnaE, which is

82 present in the dnaE gene of Nostoc punctiforme. DnaE is well characterized and has a high

83 rate of trans-splicing ${ }^{19}$. Using inteins enables strict control of toxin production, and avoids

84 toxicity due to basal expression ${ }^{14,15}$ (Supplementary Fig. 1).

85 First, we cloned full -length gyrase inhibiting toxin CcdB from Vibrio fischeri ${ }^{15}$ into a

86 plasmid (pTOX Supplementary Table 1) and transformed the toxin construct into a E. coli

87 XL2 blue (Supplementary Table 1) that constitutively expresses a genomic copy of the

88 cognate antitoxin (data not shown). We showed that $c c d B$ was bactericidal (Supplementary

89 Fig. 2) and that the intein-mediated splitting strategy led to more stable retention of the toxin-

90 harboring plasmid under repression conditions compared with a construct harboring a whole

$91 \quad c c d B$ toxin gene (Supplementary Fig. 1). We also evaluated whether three other type II

92 toxins belonging to different toxin families (ParE2, HigB2 and RelE4 ${ }^{14}$ ) could tolerate a

93 splitting and stay functional, We selected intein insertion points by inspection of 3D structure

94 predictions for toxins made in Phyre $2^{20}$, a tool for modeling protein structure (Supplementary

95 Fig. 3a). Each toxin was divided into N- and C-terminal portions (Supplementary Fig. 3b) 
96 which were fused in-frame to the $\mathrm{N}$ - or C-parts of the split intein dnaE gene (102 and 36

97 amino acids long), respectively. $\mathrm{N}$ - and C-terminal toxin-intein fusions were cloned in

98 separate, compatible plasmids ( $\mathrm{N}$ or $\mathrm{C}$ plasmids, respectively Supplementary Table 1 ) and

99 were under the control of different promoters (Fig. 1a). We validated reconstitution of the

100 active toxin by intein protein splicing in E. coli (Supplementary Fig. 4). For all five tested split

101 toxins, we found that under inducing conditions bacteria containing $\mathrm{N}$ and $\mathrm{C}$ plasmids died,

102 whereas bacteria with either the $\mathrm{N}$ or the $\mathrm{C}$ plasmid survived. $\mathrm{N}$ and $\mathrm{C}$ toxin-intein complex

103 toxicity was tested using mutations known to prevent splicing. When splicing didn't occur,

104 reconstitution of the toxin did not take place, and bacteria survived (Supplementary Fig. 4).

105 Next we chose the gyrase poison $\mathrm{CcdB}$, which is likely the most extensively

106 characterized type II toxin, to design a toxin-intein antimicrobial specific for pathogenic $V$.

107 cholerae. In $V$. cholerae one of the ToxRS-regulated genes encodes a membrane porin,

$108 \mathrm{OmpU}^{21}$. We cloned the $\mathrm{N}$ fusion of $\mathrm{CcdB}$-intein downstream of the ompU promoter

109 (regulated by ToxRS), and the $\mathrm{C}$ fusion under $\mathrm{P}_{\mathrm{BAD}}$ in the same plasmid (pU-BAD,

110 Supplementary Fig. 5a). The functionality of pU-BAD was tested in an E. coli DH5a strain

111 expressing the V. cholerae toxRS operon from a second plasmid (pRS, Supplementary Fig.

112 5a). Upon arabinose-mediated induction of toxRS expression, only bacteria containing both

113 pU-BAD and pRS plasmids died (Supplementary Fig. 5b). We replicated cell killing in

114 MG1655 (data not shown). We then tested pU-BAD activity in pathogenic $V$. cholerae strains 
$115 \mathrm{O} 1$ and $\mathrm{O} 139$ (Supplementary Fig. 6a). We observed constitutive expression of the $\mathrm{N}$-fusion

116 due to the presence of chromosomal toxRS. However, toxicity due to basal expression from

$117 \mathrm{P}_{\mathrm{BAD}}$ (Supplementary Fig. 6a) led to pU-BAD plasmid instability in V. cholerae. A V. cholerae

118 mutant lacking toxRS ( $\triangle$ toxRS) displayed normal growth and pU-BAD stability in the

119 presence of arabinose (Supplementary Fig. 6a). This suggested that $\mathrm{P}_{\text {omp }}$ could be used to

120 regulate $\mathrm{CcdB}$-intein fusion expression for targeted killing of $V$. cholerae.

121 In order to develop a conjugative CcdB-intein-based antimicrobial to specifically kill

122 pathogenic $V$. cholerae in microbial communities, we cloned a split-toxin-intein operon under

123 the control of ompU promoter in a plasmid, and added an origin of transfer (oriT) to render it

124 conjugative (plasmid pPW, Supplementary Fig. 6b, Supplementary Table 1). Conjugation is

125 carried out from donor strain E. coli $\beta 3914$, an MG1655 $\Delta$ dapA which contains the RP4

126 conjugative machinery integrated into its chromosome. pPW was introduced by conjugation

127 into $V$. cholerae strains 01,0139 and an O1- $\Delta$ toxRS mutant (Supplementary Fig. 6b), but

128 only the $\triangle$ toxRS strain was able to grow after transfer of the pPW plasmid, demonstrating

129 that it kills only Vibrio expressing ToxR.

130 We next tested whether pPW could kill specific strains in a mixed bacterial population

131 (Fig. 1b). Different recipient bacteria in this population could be distinguished in the

132 presence of X-gal: V. cholerae $\mathrm{O} 139$ (blue) and E. coli DH5 $\alpha$ (white) (Fig. 1c). We

133 conjugated $\mathrm{pPW}$ and two control plasmids (non-toxic $\mathrm{N}$ fusion containing $\mathrm{pN}_{\text {ctrl }}$ plasmid, and 
134 the pTox $_{\text {ctrl }}$ plasmid, which carries the $\mathrm{P}_{\mathrm{BAD}}$-regulated toxin-intein operon) into this mixture.

135 After conjugation of pPW from E. coli $\beta 3914$ and selection for transconjugants, pPW killed $V$.

136 cholerae $\mathrm{O} 139$ (blue bacteria) and we were only able to detect E. coli DH5otransconjugants

137 (white) on media containing XGal. Similarly, after plasmid conjugation into $V$. cholerae O1

138 and E. coli strains (MG1655), we only obtained E. coli transconjugants (Supplementary Fig.

\section{$1397 a)$.}

140 Specific killing by $\mathrm{pPW}$ relies on expression of the regulator tox $R$, which is present in

141 all Vibrio genera ${ }^{22}$. However, the ToxR regulon has evolutionarily diverged among the

142 different Vibrio species, so we analyzed pPW action in two other toxRS-containing Vibrio

143 species (Fig. 1d). We found that pPW can kill Vibrio mimicus but not Vibrio vulnificus, which

144 is more phylogenetically distant from V. cholerae, and despite harboring a ToxR ortholog,

145 does not activate ompU expression ${ }^{23}$. Additionally, we showed that our system is highly

146 specific to ToxR, since conjugation into other y-proteobacteria, such as Salmonella

147 typhimurium and Citrobacter rodentium, did not result in killing (Supplementary Fig. 7b).

148 Next we evaluated whether a split-intein toxin could kill antibiotic resistant bacteria

149 present in a community. The SXT ICE family in V. cholerae includes various antibiotic

150 resistance genes ${ }^{13}$. The SXT chassis encodes several TFs that regulate SXT transmission

151 including the SetR repressor ${ }^{13}$. We designed a module to detect SXT carriage and kill SXT-

152 harboring bacteria by implementing an additional component into our antimicrobial: the $c c d A$ 
153 gene, which encodes the antitoxin partner of $\mathrm{CcdB}$. $\operatorname{ccd} A$ was cloned downstream of the

154 SXT PL promoter, which is controlled by the SetR repressor, in a plasmid also containing the $155 c c d B$-intein operon regulated by the $\mathrm{P}_{\mathrm{BAD}}$ promoter (pPLA plasmid, Supplementary Fig. 8a,

156 Supplementary Table 1). We tested whether pPLA could kill antibiotic resistant E. coli SXT

157 (Supplementary Fig. 8b) and V. cholerae O139 (Fig. 2a). Both bacteria contain an SXT

158 element integrated at prfC. Only SXT carrying bacteria from both species were killed. All

159 bacteria lacking SXT, including V. cholerae O1 and E. coli DH5 $\boldsymbol{\alpha}$, survived (Fig. 3a and

160 Supplementary Fig. 8b). In order to develop a conjugative antimicrobial to kill antibiotic

161 resistant bacteria we added an oriT to PPLA to produce pABRW (Supplementary Table 1,

162 Fig. 2). pABRW was tested by conjugation into a mixed population of E. coli MG1655 (blue)

163 and E. coli SXT (white). Selection for pABRW yielded only E. coli MG1655 transconjugants,

164 demonstrating that pABRW specifically kills bacteria containing SXT (Fig. 2b). The same

165 result was obtained after conjugation of pABRW into $V$. cholerae 0139 mixed with $V$.

166 cholerae O1- $\Delta$ lacZ (Fig. 2c), confirming that pABRW plasmid specifically kills ABR bacteria

167 in a heterogeneous population.

168 We next combined the pPW and pABRW modules in a single plasmid. We replaced

169 the operator sequence O4 of PL with $\mathrm{O} 1$ (see online Methods) to increase SetR repression

170 to yield pFW (Supplementary Table 1, Fig. 3), which efficiently kills V. cholerae O139 (Fig.

$1713 \mathrm{3}$ ). In order to test whether non-replicative-conjugative plasmids (which would not spread 
172 toxin-intein fusions and/or antibiotic resistant genes) could harbor our killing module, we

173 changed the pSC101 replication origin to a pir-dependent R6K origin (Supplementary Fig.

174 9). R6K origin can be activated in a host expressing an ectopic pir gene in the chromosome.

175 After conjugation of pPW-R6K and pFW-R6K into bacteria that lack the pir gene, CFU/ml

176 were reduced by $60 \%$ compared with controls (Supplementary Fig. 9b). This suggests that

177 even if the plasmid cannot actively replicate once transferred in the targeted bacteria,

178 expression of the toxin is sufficient to kill these bacteria, while the use of such R6K

179 derivatives is limiting the risk of unnecessary propagation of the killing plasmid.

180 We moved onto evaluate whether our split-intein toxin could target specific bacteria

181 in natural microbiomes. We tested killing of $V$. cholerae 0139 in three niches, each of which

182 is a natural habitat for this pathogen ${ }^{24}$ : water, tropical zebrafish and a crustacean. We first

183 tested the versatility of E. coli $\beta 3914$ which is auxotrophic for the diaminopimelic acid (DAP)

184 for delivering conjugative plasmid pNctrl, in absence of DAP and found no difference in

185 conjugation rates (Supplementary Table 2). Although conjugation efficiency decreases 300

186 fold in water, $V$. cholerae transconjugants were obtained with the control plasmid $\mathrm{pN}_{\mathrm{ctrl}}$

187 (Supplementary Table 2), while using pFW, no transconjugants were detected (data not

188 shown). These results indicate that in these conditions too when receiving the pFW, $V$.

189 cholerae was killed. These preliminary data suggest that our method using pFW might hold

190 potential in bioremediation of Vibrio-contaminated water. 
192 Fig. 10a). Analysis of the microbiota composition using 16S rRNA analysis on 4 days post

193 fertilization zebrafish larvae detected less than 30 different bacterial species, mostly aerobic

194 including several Aeromonads, Pseudomonads and Stenotrophimonads (JBB and JMG,

195 unpublished). First we tested localization of both E. coli and V. cholerae, in the gut of

196 zebrafish larvae. We infected four-day-post-fertilization zebrafish larvae with fluorescently

197 tagged V. cholerae O1-GFP and E. coli-RFP. Fluorescence microscopy revealed co-

198 localization of both $V$. cholerae O1-GFP and E. coli-RFP in the digestive tract

199 (Supplementary Fig. 10a). We then tested specific killing in larvae infected with $V$. cholerae

$200 \mathrm{O} 39$ (Fig. 4a, Supplementary Fig. 11b,c). The only V. cholerae 0139 transconjugants

201 obtained were from conjugation with $\mathrm{pN}_{\text {ctrl }}$ plasmid. No V. cholerae 0139 transconjugants

202 were obtained using pFW. Therefore, pFW kills V. cholerae 0139 in zebrafish larvae (Fig. 4a

203 and Supplementary Fig. 11b). We assessed dysbiosis using observation after plating on

204 different media and didn't find any macroscopic change (Supplementary Fig. 11a). We also

205 used a mixture of 1:1 V. cholerae $\mathrm{O} 1$ and 0139 for larval infection and then infected with $E$.

206 coli $\beta 3914$ (pNctrl) or $\beta 3914$ (pFW). We detected $\mathrm{pN}_{\text {ctrl }}$ transconjugants in both $\mathrm{O} 1$ and 0139

207 serogroups, but $\mathrm{O} 1$ transconjugants only were obtained after conjugation with pFW (Fig. 4a

208 and Supplementary Fig. 11c). Therefore pFW specifically killed the 0139 serogroup. 
210 feeding and commonly found to carry various Vibrio species ${ }^{26}$ (Fig. 4b and Supplementary

211 Figs. 10b, 12). We detected co-localization of $V$. cholerae O1-GFP and E. coli-RFP in the

212 gut of $A$. salina (Supplementary Fig. 10b) and conjugation with pFW plasmid did not provoke

213 visible change in the $A$. salina microbiota, after sampling of the aerobic species on plates

214 (data not shown). Transconjugants of $V$. cholerae 0139 were only detected after

215 conjugation with $\mathrm{pN}_{\text {ctrl, }}$, but not with $\mathrm{pFW}$, showing that $\mathrm{pFW}$ kills $V$. cholerae $\mathrm{O} 139$ in $A$.

216 salina larvae (Fig. 4b and Supplementary Fig. 12a). We also infected $A$. salina with a 1:1 mix

217 of $V$. cholerae $\mathrm{O} 1$ and $\mathrm{O} 139$ and detected $\mathrm{pN}_{\text {ctrl }}$ transconjugants in $\mathrm{O} 1$ and $\mathrm{O} 139$

218 serogroups, but only detected $\mathrm{O} 1 \mathrm{pFW}$ transconjugants (Fig. 4b and Supplementary Fig.

219 12b).

220 Our split toxin-intein method can be applied to specifically kill selected bacteria

221 subtypes. We anticipate that our system could be fine-tuned to trigger toxin activation in

222 response to various environmental cues $^{27}$ including temperature, salt or $\mathrm{pH}$ by adding a

223 conditional protein splicing intein ${ }^{28}$. Inteins are functional in eukaryotic cells ${ }^{29}$, so toxin-intein

224 combinations might also be developed for targeted killing of tumor cells. The specificity of

225 our system requires identification of a species-specific transcriptional regulator, and such

226 TFs are widespread in pathogenic and antibiotic-resistant bacterial pathogens ${ }^{30}{ }^{31}$. The

227 Achilles' heel of precision antimicrobials is delivery into complex communities. Antimicrobials 
228 delivered by conjugation e.g. RNA-guided nucleases ${ }^{5}$ have reduced targeted bacterial

229 populations by 2 - to 3 -log even with a ratio of donor:recipient bacteria of $340: 1^{5}$. In our

230 experiments, using 1:1 ratios were detected a decrease in targeted bacteria ( $V$. cholerae) of

$23110 \%$, which is equivalent to the conjugation rate. We were able to kill $\approx 90-95 \%$ of the $A B R$

232 E. coli after the conjugation of pABRW by increasing ratios of donor to recipient to $10: 1$

233 (Supplementary Fig. 13). Phage delivery might be useful ${ }^{5}$, but phage have other

234 disadvantages ${ }^{32}$, including narrow host range and rapid emergence of phage resistance.

235 One advantage of our system compared with others ${ }^{4,5,33}$ is that escape mutants are less

236 frequent (below $10^{-6}-10^{-8}$; Supplementary Table 3). Analysis of escape clones

237 (Supplementary Table 4), when targeting ABR bacteria revealed that between 63 and 90\%

238 of these clones had lost the SXT element, and were not ABR (Supplementary Table 5). One

239 of the reasons for the lower chance of escape might be that toxin resistance has not been

240 observed. A different synthetic kill switch based on TA systems was also stable due to

241 minimal escape rates in vivo ${ }^{34}$. The dual regulatory system in the Final Weapon (Fig. 3a)

242 functions as an AND-logic gate, increasing effectiveness in the control of toxin production,

243 which only happens when both inputs (pathogenicity and ABR) are present. If delivery of

244 mobilizable antimicrobials can be optimized, appearance of resistant bacteria would be rare. 245 
247 REFERENCES

248 1. World Health Organization. The evolving threat of antimicrobial resistance: Options for 249 action. WHO Publ. 1-119 (2014).

250 2. Davies, J. \& Davies, D. Origins and evolution of antibiotic resistance. Microbiol. Mol.

251 Biol. Rev. 74, 417-433 (2010).

252 3. Chikindas, M. L., Weeks, R., Drider, D., Chistyakov, V. A. \& Dicks, L. M. Functions 253 and emerging applications of bacteriocins. Current Opinion in Biotechnology 49, 23$254(2018)$.

255 4. Bikard, D. et al. Exploiting CRISPR-Cas nucleases to produce sequence-specific 256 antimicrobials. Nat. Biotechnol. 32, 1146-50 (2014).

257 5. Citorik, R. J., Mimee, M. \& Lu, T. K. Sequence-specific antimicrobials using efficiently 258 delivered RNA-guided nucleases. Nat. Biotechnol. 32, 1141-1145 (2014).

259 6. Rodriguez-Pagan, I., Novick, R. P., Ross, H. F., Jiang, D. \& Ram, G. Conversion of 260 staphylococcal pathogenicity islands to CRISPR-carrying antibacterial agents that 261 cure infections in mice. Nat. Biotechnol. (2018). doi:10.1038/nbt.4203

$2627 . \quad$ Lin, D. M., Koskella, B. \& Lin, H. C. Phage therapy: An alternative to antibiotics in the age of multi-drug resistance. World J. Gastrointest. Pharmacol. Ther. 8, 162 (2017).

264 8. Jayaraman, P., Holowko, M. B., Yeoh, J. W., Lim, S. \& Poh, C. L. Repurposing a Two- 
265 Component System-Based Biosensor for the Killing of Vibrio cholerae. ACS Synth.

266 Biol. 6, 1403-1415 (2017).

267 9. Lobato-Márquez, D. et al. Toxin-antitoxins and bacterial virulence. FEMS Microbiol.

268 Rev. 40, 592-609 (2016).

269 10. Goeders, N. \& Van Melderen, L. Toxin-antitoxin systems as multilevel interaction

$270 \quad$ systems. Toxins 6, 304-324 (2013).

271 11. Ali, M., Nelson, A. R., Lopez, A. L. \& Sack, D. A. Updated global burden of cholera in

272 endemic countries. PLoS Negl. Trop. Dis. 9, 1-13 (2015).

273 12. Childers, B. M. \& Klose, K. E. Regulation of virulence in Vibrio cholerae: the ToxR

274 regulon. Future Microbiol. 2, 335-344 (2007).

275 13. Beaber, J. W., Hochhut, B. \& Waldor, M. K. SOS response promotes horizontal

276 dissemination of antibiotic resistance genes. Nature 427, 72-74 (2004).

277 14. Iqbal, N., Guérout, A. M., Krin, E., Le Roux, F. \& Mazel, D. Comprehensive functional 278 analysis of the 18 Vibrio cholerae N16961 toxin-antitoxin systems substantiates their

279 role in stabilizing the superintegron. J. Bacteriol. 197, 2150-2159 (2015).

280 15. Guérout, A. M. et al. Characterization of the phd-doc and ccd toxin-antitoxin cassettes

281 from Vibrio superintegrons. J. Bacteriol. 195, 2270-2283 (2013).

282 16. Topilina, N. I. \& Mills, K. V. Recent advances in in vivo applications of intein-mediated

283 protein splicing. Mob. DNA 5, 5 (2014). 
284 17. Li, Y. Split-inteins and their bioapplications. Biotechnology Letters 37, 2121-2137 (2015).

18. Alford, S. C., O'Sullivan, C., Obst, J., Christie, J. \& Howard, P. L. Conditional protein splicing of [small alpha]-sarcin in live cells. Mol. Biosyst. 10, 831-837 (2014).

19. Zettler, J., Schütz, V. \& Mootz, H. D. The naturally split Npu DnaE intein exhibits an extraordinarily high rate in the protein trans-splicing reaction. FEBS Lett. 583, 909-

20. Kelley, L. A., Mezulis, S., Yates, C. M., Wass, M. N. \& Sternberg, M. J. E. The Phyre2 web portal for protein modeling, prediction and analysis. Nat. Protoc. 10, 845-858 (2015).

21. Crawford, J. A., Kaper, J. B. \& DiRita, V. J. Analysis of ToxR-dependent transcription activation of $\mathrm{ompU}$, the gene encoding a major envelope protein in Vibrio cholerae. Mol. Microbiol. 29, 235-246 (1998).

22. Osorio, C. R. \& Klose, K. E. A region of the transmembrane regulatory protein ToxR that tethers the transcriptional activation domain to the cytoplasmic membrane displays wide divergence among vibrio species. J. Bacteriol. 182, 526-528 (2000).

23. Lee, S. E. et al. Vibrio vulnificus has the transmembrane transcription activator ToxRS (2000). 
303 24. Vezzulli, L., Pruzzo, C., Huq, A. \& Colwell, R. R. Environmental reservoirs of Vibrio

304 cholerae and their role in cholera. Environmental Microbiology Reports 2, 27-33

$305 \quad$ (2010).

306 25. Runft, D. L. et al. Zebrafish as a natural host model for Vibrio cholerae colonization and transmission. Appl. Environ. Microbiol. 80, 1710-1717 (2014). Environ. Microbiol. 7, 1488-1495 (2005).

26. Austin, B., Austin, D., Sutherland, R., Thompson, F. \& Swings, J. Pathogenicity of vibrios to rainbow trout (Oncorhynchus mykiss, Walbaum) and Artemia nauplii.

27. Lennon, C. W. \& Belfort, M. Inteins. Current Biology 27, R204-R206 (2017).

28. Callahan, B. P., Topilina, N. I., Stanger, M. J., Van Roey, P. \& Belfort, M. Structure of catalytically competent intein caught in a redox trap with functional and evolutionary implications. Nat. Struct. Mol. Biol. (2011). doi:10.1038/nsmb.2041

29. Zhu, F. X. et al. Inter-chain disulfide bond improved protein trans-splicing increases plasma coagulation activity in C57BL/6 mice following portal vein FVIII gene delivery by dual vectors. Sci. China Life Sci. 56, 262-267 (2013).

30. Hacker, J. \& Kaper, J. B. PATHOGENICITY ISLANDS AND THE EVOLUTION OF MICROBES. Annu. Rev. Microbiol 54, 641-79 (2000).

31. Main-Hester, K. L., Colpitts, K. M., Thomas, G. A., Fang, F. C. \& Libby, S. J. 
324 32. Loc-Carrillo, C. \& Abedon, S. Pros and cons of phage therapy. Bacteriophage 1, 111$114(2011)$.

33. Yosef, I., Manor, M., Kiro, R. \& Qimron, U. Temperate and lytic bacteriophages programmed to sensitize and kill antibiotic-resistant bacteria. Proc. Natl. Acad. Sci. 112, 7267-7272 (2015). Cell 68, 686-696 (2017). 
334 ACKNOWLEDGMENTS

335 We would like to thank E. Krin and M. Gugger for providing chromosome DNA for $V$.

336 cholerae and V. fischeri, and $N$. punctiforme cells, respectively and V. Burrus for V. cholerae

337 O139. We thank G. Cambray and Z. Baharoglu for V. cholerae-GFP strain and RFP-

338 containing plasmid, respectively. We thank S. Jin for her technical help. We thank also P.

339 Escoll for assistance with microscopy, Valerie Briolat for providing us with the zebrafish and

340 Artemias, A. Gomez-Losada for his help with the statistics treatment, and S. Aguilar-Pierlé

341 for helpful reading of the manuscript. We thank F. de la Cruz for his invaluable comments

342 along the development of this work. This work was supported by the Institut Pasteur (D.M.

343 and J.-M.G. Units), the Centre National de la Recherche Scientifique (CNRS-UMR 3525)

344 (D.M.), PLASWIRES 612146/FP7- FET-Proactive (D.M., A.R.-P Lab, R.L.-I. salary), the

345 French Government's Investissement d'Avenir program, Laboratoire d'Excellence

346 "Integrative Biology of Emerging Infectious Diseases" (grant nANR-10-LABX-62-IBEID to D.

347 M. and J.-M.G. Units), Spanish project TIN2016-81079-R (AEI/FEDER, EU) and Comunidad

348 de Madrid (cofound with FES and FEDER, EU) B2017/BMD-3691 project ingeMICS-CM

349 (A.R.-P), the Fondation pour la Recherche Médicale (Grant No. DBF20160635736 to D.M.

350 and DEQ20140329508 to J.-M.G.). J.B.-B. was the recipient of a long-term post-doctoral

351 fellowship from the Federation of European Biochemical Societies (FEBS). 


\section{AUTHOR CONTRIBUTIONS}

354 D.M. and R.L.-I designed the experiments. J.B.-B and R.L.-I designed and performed the in

355 vivo experiments. J.B.-B. performed the microscopy experiments and statistic analysis.

356 D.M., R.L.-I and A.R.-P participated in the conception of the project. R.L.-I and D.M.

357 prepared the manuscript and wrote the article with large participation of J.B.-B, J.-M.G. and

358 A.R.-P.

359

360 COMPETING FINANCIAL INTERESTS

361 The authors declare no competing financial interests.

362

363

DATA AVAILABILITY STATEMENT

364 The data, plasmids and strains generated for this study, that support our findings are

365 available upon request to the corresponding author.

366 


\section{Figure Legends:}

368 Figure 1. Specific killing of pathogenic $V$. cholerae in mixed population of 369 bacteria mediated by toxin-intein strategy. (a) Schematic representation of the 370 active toxin production from plasmids encoding split toxin (red) combined with split

371 intein (blue) inside a bacterium. The first half of the toxin is fused with N-terminal split 372 intein gene ( $\mathrm{N}$ plasmid) under the control of $\mathrm{P}_{\mathrm{BAD}}$ promoter and the second half of the 373 toxin is fused with the C-terminal intein gene ( $\mathrm{C}$ plasmid) and it is controlled by PLaC.

374 Expression of these fusions is activated by the addition of arabinose and IPTG, 375 respectively. Recognition of the protein fusions takes place by the intein module, 376 which carry out the splicing process, which lead to toxin reconstitution, provoking cell 377 death. (b) Mode of action of the genetic weapon spreading through conjugation in 378 mixed population of bacteria and killing of targeted harmful bacteria. (c) Mixed 379 population of $V$. cholerae $\mathrm{O} 139$ (blue) and E. coli DH5a (white) as recipients for 380 conjugation using $\beta 3914$ as donor strain containing $\mathrm{pN}_{\mathrm{ctrl}}$, $\mathrm{pTox_{ctrl }}$ or $\mathrm{pPW}$ plasmids.

381 The ompU promoter activated specifically by ToxRS from $V$. cholerae is represented 382 by a circled (+) pink-symbol. Transconjugants were selected in $\mathrm{MH}+$ Spectinomycin 383 (Sp), X-gal for color development and arabinose for induction of PBAD. (d)

384 Conjugation of $\mathrm{pN}_{\mathrm{ctrl}}$, $\mathrm{pTox}_{\mathrm{ctrl}}$ and $\mathrm{pPW}$ plasmid using 33914 as donor strain in Vibrio 385 mimicus and Vibrio vulnificus. Transconjugants were selected in $\mathrm{MH}$ media $+\mathrm{Sp}$ and 386 arabinose for induction of $\mathrm{P}_{\mathrm{BAD}}$. Pictures are representative of three independent 387 experiments.

390 Figure 2. Specific killing of antibiotic resistant bacteria (containing SXT). (a)

391 pPLA plasmid that contains CcdB-intein fusion operon under $\mathrm{P}_{\mathrm{BAD}}$ control expression 
392 and $c c d A$ antitoxin under PL promoter (symbolized by an orange circled -) which is

393 repressed by SetR. Growth test of $V$. cholerae 01 or 0139 containing PPLA plasmid

394 in MH media $+\mathrm{Sp}$ and supplemented with glucose (GLU) or arabinose (ARA). (b)

395 Mixed population of E. coli MG1655 (blue) and E. coli SXT (white) as recipients for

396 conjugation using $\beta 3914$ as donor strain containing $\mathrm{pN}_{\text {ctrl, }}$ pTox $\mathrm{x}_{\text {ctrl }}$ or $\mathrm{pABRW}$

397 plasmids. Transconjugants were selected on $\mathrm{MH}+\mathrm{Sp}, \mathrm{X}$-gal for species

398 identification and arabinose for induction of $\mathrm{P}_{\mathrm{BAD}}$. (c) Mixed population of $V$. cholerae

399 O139-SXT (blue) and V. cholerae O1 (white) as recipients for conjugation using

$400 \quad \beta 3914$ as donor strain containing $\mathrm{pN}_{\mathrm{ctrl}}, \mathrm{p}$ Tox $\mathrm{x}_{\mathrm{ctrl}}$ or $\mathrm{pABRW}$ plasmids as described in

401 b). Pictures are representative of three independent experiments.

404 Figure 3. Design, tuning and assay of the final weapon pFW, obtained by 405 putting together the pathogenicity and antibiotic resistance (ABR) modules in 406 a single conjugative vector. (a) Schematic representation of the specific killing of $407 \quad V$. cholerae $\mathrm{O} 139$ after pFW conjugation (left). Schematic display of the 408 corresponding AND-logic gate (right). (b) Conjugation from $\beta 3914$ of either $\mathrm{pN}_{\text {ctrl }}$ or $409 \mathrm{pFW}$, of $V$. cholerae serogroup $\mathrm{O} 139$ (blue) and $\mathrm{O} 1$ (white) as recipient mixed

410 population. Transconjugants were selected on $\mathrm{MH}+\mathrm{Sp}$ (plasmid marker). pFW

411 plasmid was obtained after change in RBS sequence of $o m p U$ promoter to increase

412 translation of toxin-intein fusion and substitution of the $\mathrm{O} 4$ operator sequence by $\mathrm{O} 1$

413 operator sequence (see online methods) to increase SetR binding affinity to the PL

414 promoter, and consequently increase repression. Only V. cholerae serogroup $\mathrm{O} 1$

415 that is devoid of SXT in its genome was detected after pFW conjugation,

416 demonstrating the specific killing of serogroup 0139, which contain both

417 chromosomally encoded ToxR and SetR the chosen indicators of pathogenicity and 
418 antibiotic resistance, respectively. Pictures are representative from three

419 independent experiments.

\section{Figure 4. Specific killing of pathogenic and ABR V. cholerae 0139 in the}

zebrafish larvae and Artemia salina nauplii models. (a) Four-day-postfertilization zebrafish larvae were exposed to water containing $10^{4} \mathrm{CFU} / \mathrm{ml} V$. cholerae 0139 or a mixed population containing $10^{5} \mathrm{CFU} / \mathrm{ml} V$. cholerae $0139+V$. cholerae $\mathrm{O} 1$, and then infected (see methods) with $10^{7}$ (O139) or $10^{6}$ (mix Vibrio) CFU/ml of $\beta 3914$ as donor strain of either $\mathrm{pN}_{\mathrm{ctrl}}$ or $\mathrm{pFW}$ plasmids. Five larvae were fished and mashed to analyze its microbiota. Transconjugants were selected in $\mathrm{MH}$ media with $\mathrm{Sp}$ and $\mathrm{X}$ gal. Transconjugants were only detected after conjugation with $\mathrm{pN}_{\text {ctr }}$ plasmid for $\mathrm{O} 139$ and not after pFW conjugation as expected from the specific killing. Confirming 431 pFW specifity, pFW transconjugants were detected for V. cholerae O1, which should 432 not be killed by this plasmid. Data for $\mathrm{O} 139$ represent transconjugants obtained from 43315 larvae fished in three independent experiments $(n=3$, mean $\pm s . d)$, and data from 434 the mix of Vibrio represent transconjugants obtained from 10 larvae in two 435 independent experiments $(n=2$, mean \pm s.d). (b) Artemia salina stage nauplii were 436 infected with $10^{7} \mathrm{CFU} / \mathrm{ml} V$. cholerae 0139 or a mix of $10^{7} \mathrm{CFU} / \mathrm{ml}$ V. cholerae 0139 $437+V$. cholerae 01 (see methods). Then exposed to $10^{7} \mathrm{CFU} / \mathrm{ml} \beta 3914$ as donor strain of either $\mathrm{pN}_{\mathrm{ctrl}}$ or $\mathrm{pFW}$ plasmids. Transconjugants were selected in $\mathrm{MH}$ media with $\mathrm{Sp}$ and X-gal. As in zebrafish, transconjugants were only detected after conjugation with $\mathrm{pN}_{\mathrm{ctrl}}$ plasmid for $\mathrm{O} 139$ and not after $\mathrm{pFW}$ conjugation. As expected, $V$.

441 cholerae $\mathrm{O} 1 \mathrm{pFW}$ transconjugants were also detected in this in vivo model. Data 442 numbers were calculated from four independent experiments $(n=4$, mean $\pm s . d)$. 


\section{ONLINE METHODS}

2

3 Strains and culture conditions.

4 Unless otherwise noted, bacterial cultures were grown at $37^{\circ} \mathrm{C}$ with Luria-Bertani (LB)

5 medium (Lennox) or Mueller-Hinton $(\mathrm{MH})$ solid media supplemented when appropriate,

6 with the following antibiotics: $50 \mu \mathrm{g} / \mathrm{ml}$ kanamycin (Kan), $50 \mu \mathrm{g} / \mathrm{ml}$ chloramphenicol

$7 \quad(\mathrm{Cm}), 100 \mu \mathrm{g} / \mathrm{ml}$ carbenicillin (Carb), 50 or $100 \mu \mathrm{g} / \mathrm{ml}$ spectinomycin (Sp) for E. coli and

$8100 \mu \mathrm{g} / \mathrm{ml} \mathrm{Sp}$ for Vibrio cholerae. Selection of transconjugants was carried using 100

$9 \mu \mathrm{g} / \mathrm{ml} \mathrm{Sp}$ in all cases, except for $V$. mimicus and $V$. vulnificus where we used $50 \mu \mathrm{g} / \mathrm{ml}$

10 Sp. Bacterial strains used in this study are listed in Supplementary Table 1. Other

11 molecules were added to the media with the following concentrations: $40 \mu \mathrm{g} / \mathrm{ml} \mathrm{5-}$

12 bromo-4-chloro-3-indolyl-beta-D-galactopyranoside (Xgal), $0.3 \mathrm{mM}$ Diaminopimelic acid

13 (DAP), 1\% glucose and $0,2 \%$ arabinose.

14

15 Plasmid construction.

16 Plasmids are listed in Supplementary Table 1 and primers in Supplementary Table 6.

17 All plasmid sequences were verified through sequencing.

18 To generate the $\mathrm{N}$ and $\mathrm{C}$ plasmids for each toxin-intein fusion, the $\mathrm{N}$ - and $\mathrm{C}$-terminal

19 toxin regions were amplified with primers F-toxin-EcoRl/R-toxin-intein and F-toxin-

20 intein/R-toxin-Xbal, respectively. $\mathrm{N}$-and $\mathrm{C}$-terminal intein regions were amplified with

21 primers F-intein-toxin/R-intein-Xbal and F-intein-EcoRI/R-intein-toxin, respectively. As

22 DNA templates for toxins we used chromosomal DNA from $V$. cholerae in all cases and

23 V. fischeri for $c c d B$. Intein amplification was done with chromosomal DNA from the

24 cyanobacteria Nostoc punctiforme. PCR products of $\mathrm{N}$ - and C-terminal regions were

25 fused by Gibson assembly ${ }^{35}$. Each toxin-intein fusion was then digested with

$26 \mathrm{EcoRI} / \mathrm{Xbal}$ (Thermo Fisher) and then cloned in EcoRI/Xbal digested $\mathrm{pBAD} 43^{36}$ and

$27 \mathrm{pSU}^{37} 8^{37}$ (or pSU18) plasmids, respectively (Supplementary Table 1). To generate the 
mutated version of $\mathrm{N}$-terminal plasmid $\left(\mathrm{n}^{\star}\right)$ whole plasmids were amplified using 29 primers F-Int-tox-mut/R-int-tox-mut.

30 To assemble the pU-BAD plasmid (Supplementary Fig. 5) we first cloned the C31 terminal CcdB-Npu fusion into a pBAD18 plasmid (EcoRI-Xbal). An ompU promoter 32 was inserted upstream the $\mathrm{N}$-terminal $\operatorname{ccdB} / \mathrm{Npu}$ fusion in $\mathrm{N}$ plasmid by PCR. The 33 ompU promoter region was amplified using F-PompU-1/R-PompU-dB (size=352 bp).

34 This promoter was chosen based on previous work $^{21}$ that showed its high induction in 35 the presence of ToxR. A region containing the pSC101 origin was amplified using R36 BAD43-BAD18/F-4126 primers and the N-terminal CcdB-Npu plasmid as template. A 37 second region containing the N-terminal fusion was amplified using 4217/R-BAD4338 BAD18 primers and $\mathrm{N}$-terminal plasmids also as templates. Other regions containing 39 the C-terminal fusion and Kan resistance gene were amplified using R-BAD1840 BAD43/F-BAD18-BAD43 primers and the C-terminal CcdB-Npu pBAD18 plasmid as 41 template. PCR products were then fused by Gibson assembly ${ }^{35}$ producing the pU-BAD 42 plasmid.

43 To generate the pRS plasmid, (Supplementary Fig. 5) the toxRS operon from $V$. 44 cholerae 01 was amplified using F-toxR-Sacl/R-toxS-Xbal primers, digested with Sacl 45 and Xbal and ligated with Sacl-Xbal digested plasmid pBAD30. The native RBS 46 sequence of tox $R$ was kept.

47 To assemble the toxin-intein $\mathrm{N}$ and C-terminal fusions as an operon (pToxInt plasmid), $48 \mathrm{~N}$ - and C-fusions were amplified using F-CcdB-EcoRI/R-Int-N-Int-C and F-Int-C-Int49 N/R-Int-Xbal primers and then ligated by Gibson assembly ${ }^{35}$, digested with EcoRI/Xbal 50 and cloned into a pBAD43-EcoRI/Xbal digested plasmid. The fusion contains the 51 following sequence: 5' TGATAAGGAGGTAACATATG 3' between the $\mathrm{N}$ and $\mathrm{C}$ genes. 52 This sequence contains the RBS sequence necessary for translation of the C-terminal 53 fusion. The pTox plasmid was created by amplification of the $\operatorname{ccdB}$ toxin gene from $V$. 54 fischeri DNA with F-CcdB-EcoRI/R-CcdB-Xbal primers, EcoRI/Xbal digestion and 55 ligation into a pBAD43-EcoRI/Xbal digested plasmid. E. coli XL2blue strain that 
contains $F^{\prime}$ plasmid integrated in the chromosome (containing the $\operatorname{ccdB} / \mathrm{ccdA}$ TA

57 system and conferring resistance to $\mathrm{CcdB}$ ), was used to transform with this ligation in 58 order to obtain positive clones.

59 To assemble the pPW genetic weapon, the ompU promoter was amplified as 60 previously described and ligated by Gibson assembly ${ }^{35}$ with the product of pToxInt 61 plasmid PCR using F-dB-PompU/R-BAD-PU1 primers.

62 The pPLA plasmid was constructed first by amplifying by PCR the PL promoter ${ }^{38}$ using 63 DNA from $V$. cholerae 0139 and F-PL-plasmid/R-PL-ccdA as primers. Then, the ccdA antitoxin gene was amplified using the F-ccdA-PL/R-ccdA-plasmid primers and $V$. 65 fischeri DNA. Finally, the pTox-Int plasmid was also amplified using F-plasmid-dA/R66 plasmid-PL primers. Ligation by Gibson assembly ${ }^{35}$ of the three PCR products resulted 67 in the PPLA plasmid.

68 Mobilizable genetic weapons were created by amplifying the origin of transfer oriT RP4 69 using F-pSW23-BAD/R-oriT-BAD43 primers and the plasmid $\mathrm{pSW}^{2} \mathrm{~T}^{39}$ as template. 70 Then, the oriT PCR product was ligated through Gibson assembly ${ }^{35}$ with the amplified 71 plasmid using F-BAD-pSW/R-BAD43-oriT primers and the weapon or control plasmids 72 as template.

73 To assemble the Final Weapon we the plasmid pFW (Figure 3) as follow. The ompU 74 promoter-1 was ligated into the pABRW plasmid as previously described for the pU75 BAD construction. In order to fine-tune the RBS of ompU in this plasmid as well as the 76 PL promoters,, PCRs were performed using F-ccdB-SD-OK/R-PU-SD-OK and F-PL77 SD-T/R-PL-SD-T primer pairs, respectively. Finally, to generate the pFW plasmid, an 78 operator $\mathrm{O} 1$ sequence $\left(\right.$ see $^{38}$ ) was added into the PL promoter by PCR amplification of 79 the pFW2 plasmid using F-PL-O1/R-PL-O1 primers.

80 To generate the pPW-R6K, pFW-R6K and pNctrl-R6K plasmids we first amplified the 81 R6K replication origin using F-R6K-weapon/R-R6K-weapon primers and the pMP7 ${ }^{40}$ 82 plasmid as template. Then, the pPW, pFW and pNctrl plasmids were amplified using F- 
weapon-R6K/R-weapon-R6K primers. Finally, PCR fragments were ligated by Gibson assembly ${ }^{35}$.

\section{$\Delta$ toxRS strain construction}

DNA regions 500 bp upstream and downstream of the toxRS operon were amplified using F-toxRup-p7/R-toxRups and F-toxSdow/R-toxSdow-p7, respectively. The amplified fragments were ligated by Gibson assembly ${ }^{35}$ and then cloned into an R6K Yori-based suicide vector, pSW7848 ${ }^{40}$ that encodes the $c c d B$ toxin gene under the control of an arabinose-inducible promoter, $\mathrm{P}_{\mathrm{BAD}}$. For conjugal transfer of plasmids into V. cholerae strains, E. coli $\beta 3914$ was used as the donor. Clones where integration of the entire plasmid in the chromosome by single crossover occurred were selected. Elimination of the plasmid backbone resulting from a second recombination step was selected as described ref 39 .

\section{Transformation assays}

DH5a chimiocompetent cells (Invitrogen) were transformed with 150 ng of pTox, pToxInt or pN plasmids (Supplementary Fig. 1a). Transformants were then tested in Sp containing media with glucose or arabinose to analyze toxin integrity. 10 to $12 \%$ of pTox-transformed clones from were able to grow in the presence of arabinose. Four independent clones were analyzed by sequencing and they all carried an insertion sequence in the $c c d B$ toxin gene. These clones were responsible for pTox transformation rate decrease in comparison with the $\mathrm{pToxInt}$ and $\mathrm{pN}$ plasmids.

DH5a cells (Invitrogen) were co-transformed with two plasmids simultaneously. Both plasmids were then simultaneously selected (Supplementary Fig.4).

Transformation of the donor strain $\beta 3914$ was performed in the presence of DAP.

\section{Growth tests}


110 Eighteen independent clones from $\mathrm{DH} 5 \alpha$ transformation were inoculated in p96

111 microplates containing LB media with $\mathrm{Sp}$ and glucose. The TECAN Infinite 200

112 microplate reader (TECAN, Männedorf, Germany) was used to determine growth

113 curves, with absorbance $(620 \mathrm{~nm})$ taken at 6-minute intervals for a period of $12 \mathrm{~h}$. The

114 obtained OD values were plotted as seen on Supplementary Fig. $1 \mathrm{~b}$.

115 In Supplementary Fig. 2 for analysis of bactericide effect of CcdB toxin: V. cholerae

1160139 was co-transformed with antitoxin-ccdA (pBAD24-ccdA) and pPW plasmids in

117 the presence of arabinose allowing the antitoxin to be expressed. pPW plasmid

118 contains the toxin-intein under the control of $\mathrm{ompU}$ promoter, which is always active in

119 V. cholerae. Bacteria culture supplemented with antibiotics for maintaining both

120 plasmids and arabinose, were diluted at $\mathrm{OD}=0.5$ (time $0 \mathrm{~h}$ ). Then bacteria were washed

121 three times with $\mathrm{MH}$ media with antibiotics and glucose, in order to switch off antitoxin

122 expression, and incubated for $4 \mathrm{~h}$ at $37^{\circ} \mathrm{C}$. Total bacteria were calculated by the $\mathrm{CFU} / \mathrm{ml}$

123 at time $0 \mathrm{~h}$ and $4 \mathrm{~h}$ present in $\mathrm{MH}$ media with antibiotics and with glucose (1\%) or

124 arabinose $(0,2 \%)$. Data numbers were calculated from four independent experiments $125(n=4)$.

126

\section{Conjugation assays.}

128 Overnight cultures of donor and recipient strains were diluted 1:100 in culture media 129 with antibiotic and grown at $37^{\circ} \mathrm{C}$ for $2-3$ hours. Then, cultures were diluted to an $\mathrm{OD}_{600}$

$130=0.5$. The different conjugation experiments were performed by a filter mating 131 procedure described previously ${ }^{41}$ with a donor/recipient ratio of $1:: 1$. When the 132 recipients were composed of a mixed population the donor/mixed-recipient ratio was

$1331:: 0.5-0.5$. Before mixing the different bacteria, cultures were washed three times with 134 fresh media to remove antibiotics. In Supplementary Table 2 bacteria were mixed in 135 different proportions (2:1 and 3:1) to test whether this would impact conjugation 136 efficiency. Conjugation was performed during $4 \mathrm{~h}$ at $37^{\circ} \mathrm{C}$ on filter in $\mathrm{MH}$ plates 
137 supplemented with DAP (and containing $\mathrm{NaCl}$ until $332 \mathrm{mM}$ final concentration in the 138 case of $V$. vulnificus).

139

140 In vivo conjugation in zebrafish larvae and Artemia salina

141 All animal experiments described in the present study were conducted at the Institut

142 Pasteur according to European Union guidelines for handling of laboratory animals

143 (http://ec.europa.eu/environment/chemicals/lab_animals/home_en.htm) and were

144 approved by the Institut Pasteur Animal Care and Use Committee and the Direction

145 Sanitaire et Veterinaire de Paris under permit \#A-75-1061. Conjugative killing was

146 assessed as follow. Four-day-postfertilization zebrafish larvae were exposed to water

147 containing $10^{4} \mathrm{CFU} / \mathrm{ml}$ of $V$. cholerae 0139 for 2 hours at $27^{\circ} \mathrm{C}$ (Figure $4 \mathrm{a}$ ) or a $1:: 1$

148 mixed population containing $10^{5} \mathrm{CFU} / \mathrm{ml} \mathrm{V}$. cholerae $0139+$ V. cholerae $\mathrm{O} 1$ (Figure

$1495 \mathrm{a}$, mix Vibrio) for 2 hours at $27^{\circ} \mathrm{C}$. Then, larvae were washed in sterile water three 150 times and then placed into a well containing $10^{7}$ or $10^{6} \mathrm{CFU} / \mathrm{ml}$ (Figure $4 \mathrm{a}, \mathrm{V}$. cholerae

1510139 and mix Vibrio, respectively) of the E. coli $\beta 3914-\Delta$ dap donor strain containing

152 either the $\mathrm{pN}_{\text {ctrl }}$ or $\mathrm{pFW}$ plasmid for 24 hours at $27^{\circ} \mathrm{C}$. In Supplementary Fig. $11 \mathrm{~b}$ and

153 11c, infection dose for Vibrio was the same than for Fig. 4a. Larvae were transferred to

154 bacteria-free wells, washed in sterile water three times and then placed into a well

155 containing Tricaine (Sigma-Aldrich \#E10521) at $200 \mathrm{mg} / \mathrm{ml}$ to euthanize them. Finally,

156 they were transferred to a tube containing calibrated glass beads (acid washed, 425

157 um to $600 \mathrm{um}$, Sigma-Aldrich \#G8722) and $500 \mu \mathrm{l}$ of water. Five larvae were mashed

158 using FastPrep ${ }^{\circledR}$ Cell Disrupter (BIO101/FP120 QBioGene) for 45 seconds at

159 maximum speed $(6,5 \mathrm{~m} / \mathrm{sec})$ to analyze their microbiota (Supplementary Fig. 11) in $\mathrm{MH}$

160 Media + X-gal or TCBS media for selection of $V$. cholerae. Blue bacteria corresponding

161 to $V$. cholerae 0139 were detected in $\mathrm{MH}$ media. Transconjugants selection was done

162 into $\mathrm{MH}$ Media $+\mathrm{X}$-gal and Sp and then, replication of these $\mathrm{MH}$ plates were done on

163 TCBS media to specific identify $V$. cholerae. Strain identity was confirmed through 
164 yellow color development in TCBS Vibrio specific media. The amoeba Tetrahymena

165 thermophila (T. thermophila) was added to feed larvae during the experiment.

166

167 Groups of $225 \pm 15$ larvaes of Artemia salina stage nauplii suspended in $1 \mathrm{ml}$ volume of 168 seawater were washed using sterile cell strainer Nylon filters $100 \mu \mathrm{m}$ pore size 169 (Falcon) and three times with the same volume $(3 \times 1 \mathrm{ml})$ of sterile PBS (D8537, Sigma).

170 Nauplii were suspended in $1 \mathrm{ml}$ PBS and then infected with $10^{7} \mathrm{~V}$. cholerae 01 or a mix 171 of $10^{7} \mathrm{~V}$. cholerae $\mathrm{O} 1$ and 0139 for 2 hours in agitation at $27^{\circ} \mathrm{C}$. Then nauplii were 172 washed as previously described and exposed to $10^{7}$ of $\beta 3914-\Delta$ dap bacteria with $173 \mathrm{pN}_{\mathrm{ctrl}}$ or $\mathrm{pFW}$ plasmid for 4 hours at $27^{\circ} \mathrm{C}$. These experiments were repeated four times 174 independently. The microbiota from $1 \mathrm{ml}$ containing $225 \pm 15$ nauplii were analyzed as 175 previously described for zebrafish. In the case of Artemia, we have used M63B1 176 minimal media where Artemia feel asleep and then put them on ice, previous the use of 177 fast-prep (FastPrep ${ }^{\circledR}$ Cell Disrupter (BIO101/FP120 QBioGene) for 45 seconds at 178 maximum speed $(6,5 \mathrm{~m} / \mathrm{sec}))$. Transconjugants were selected from $225 \pm 15$ nauplii 179 after $\mathrm{pN}_{\text {ctrl }}$ or $\mathrm{pFW}$ conjugation treatment into $\mathrm{MH}$ media with $\mathrm{Sp}$ and $\mathrm{X}$-gal 180 (Supplementary Fig. 12a,b). For the identification of $V$. cholerae in the mix of both 181 serogroups (Fig. 4b and Supplementary Fig. 12b), replication of these MH plates were 182 done into TCBS media to specificaly identify $V$. cholerae. Strain identity was confirmed 183 through yellow color development in TCBS Vibrio specific media.

185 Co-localization of $E$. coli and $V$. cholerae in the zebrafish larvae and $A$. salina by 186 Microscopy.

187 Co-localization of $E$. coli and $V$. cholerae in the zebrafish larvae was assessed as 188 follow. Four-day-postfertilization zebrafish larvae were exposed to water containing $10^{6}$ $189 \mathrm{CFU} / \mathrm{ml}$ V. cholerae O1-GFP for 2 hours at $27^{\circ} \mathrm{C}$. Then washed in sterile water three 190 times and then placed into a well containing $10^{7} \mathrm{CFU} / \mathrm{ml}$ of E. coli-RFP for 24 hours at $19127^{\circ} \mathrm{C}$. Larvae were removed from the well and then placed into a well containing 
192 Tricaine for euthanize them. Infected and non-infected larvae were visualized by 193 fluorescence microscopy (EVOS FL microscope-Life technologies) using appropriate 194 wavelength conditions enabling or not the visualization of GFP and RFP. Fluorescence 195 was only detected in infected larvae and more precisely into the gut where both 196 bacteria are co-localized.

197 In the case of $A$. salina stage nauplii the microscopy experiment was done using $10^{7} \mathrm{~V}$. 198 cholerae-GFP for 2 hours in agitation at $27^{\circ} \mathrm{C}$. Then nauplii were washed as previously 199 described and exposed to $10^{7}$ of E. coli-RFP strain for 2 hours. Microscopy conditions 200 were performed as for zebrafish experiment.

201

\section{Statistics}

203 In Supplementary Fig. 9, one-way ANOVA with Dunnett's Multiple Comparison Test 204 was performed. PNcontrol-R6K vs pPW-R6K, Mean Diff. $=2.383 e+008, q=4.183$, $205{ }^{* *} \mathrm{P}<0.05,95 \% \mathrm{Cl}$ of diff $=(8.937 \mathrm{e}+007$ to $3.871 \mathrm{e}+008)$. PNcontrol-R6K vs pFW-R6K, 206 Mean Diff. $=2.308 \mathrm{e}+008, q=4.227,{ }^{\star *} \mathrm{P}<0.05,95 \% \mathrm{Cl}$ of diff $=(9.187 \mathrm{e}+007$ to $2073.896 e+008)$.

208 In Supplementary Fig. 13, one-sided t-test Mann Withney was performed. E. coli SXT 209 vs $E$. coli MG1655. $\mathrm{P}$ value $=0.0143 .{ }^{*} \mathrm{P}<0.05$

210

\section{References}

212

213 35. Gibson, D. G. et al. Enzymatic assembly of DNA molecules up to several

214 hundred kilobases. Nat. Methods 6, 343-345 (2009).

215 36. Guzman, L. M., Belin, D., Carson, M. J. \& Beckwith, J. Tight regulation, 216 modulation, and high-level expression by vectors containing the arabinose $217 \quad \mathrm{P}(\mathrm{BAD})$ promoter. J. Bacteriol. 177, 4121-4130 (1995).

218 37. Bartolomé, B., Jubete, Y., Martínez, E. \& de la Cruz, F. Construction and 219 properties of a family of pACYC184-derived cloning vectors compatible with 
pBR322 and its derivatives. Gene 102, 75-78 (1991).

38. Poulin-Laprade, D. \& Burrus, V. A y Cro-like repressor is essential for the induction of conjugative transfer of SXT/R391 elements in response to DNA damage. J. Bacteriol. 197, 3822-3833 (2015).

224 39. Demarre, G. et al. A new family of mobilizable suicide plasmids based on broad host range R388 plasmid (IncW) and RP4 plasmid (IncPa) conjugative machineries and their cognate Escherichia coli host strains. Res. Microbiol. 156, 245-255 (2005).

40. Val, M. E., Skovgaard, O., Ducos-Galand, M., Bland, M. J. \& Mazel, D. Genome engineering in Vibrio cholerae: A feasible approach to address biological issues. PLoS Genet. 8, (2012).

231 41. Biskri, L., Bouvier, M., Guérout, A., Boisnard, S. \& Mazel, D. Comparative study 232 of class 1 integron and Vibrio cholerae superintegron integrase activities. $J$ Bacteriol 187, 1740-1750 (2005). 
a

b

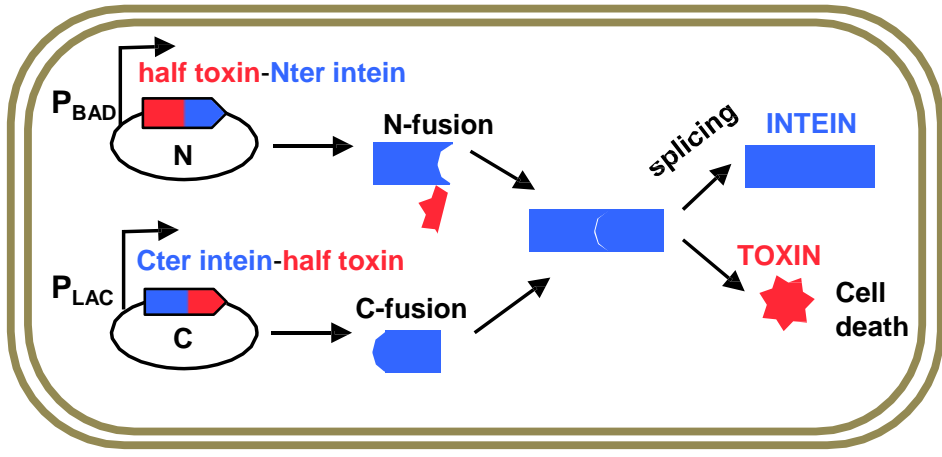

C

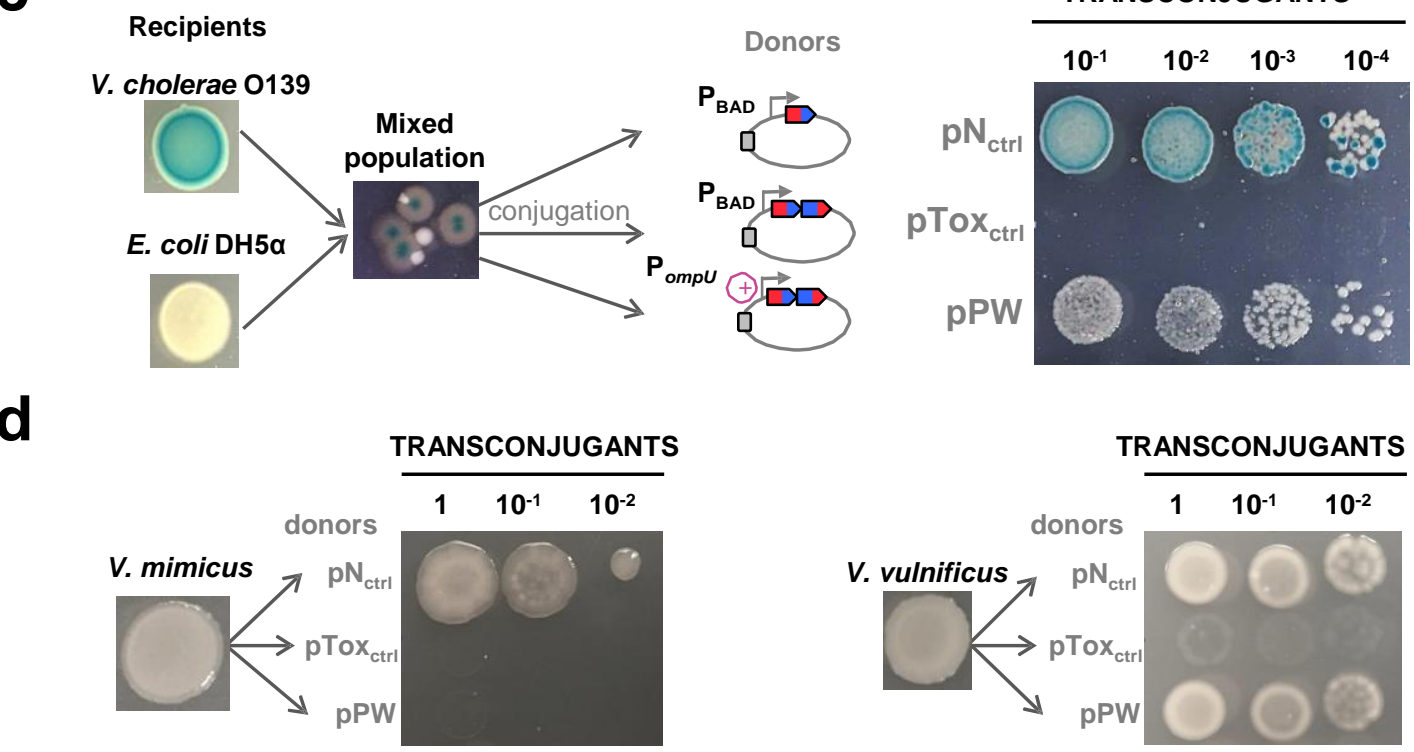

TRANSCONJUGANTS

Figure 1 
a

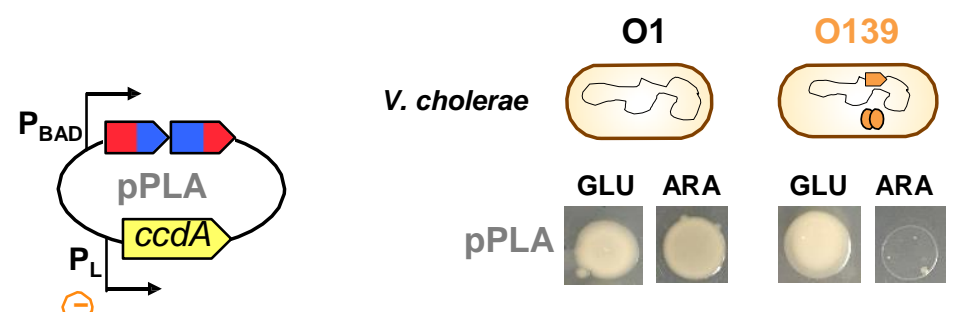

b

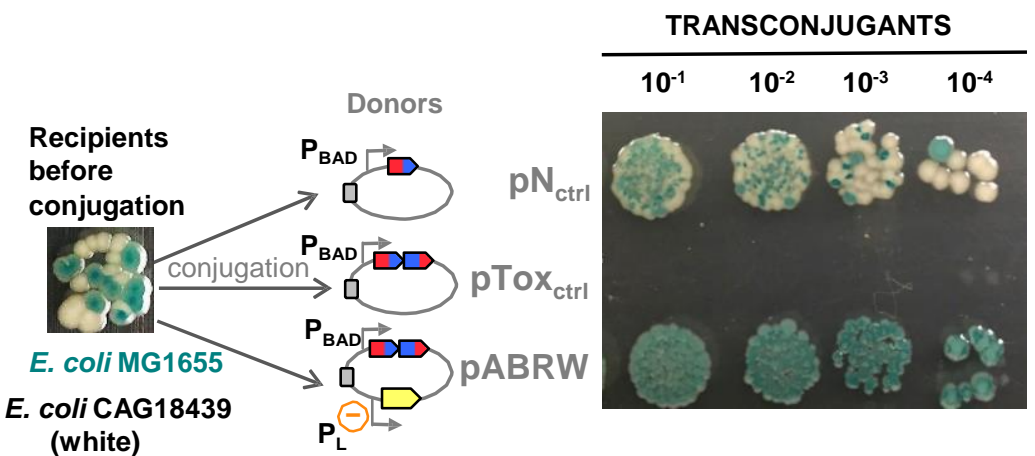

C (white)

TRANSCONJUGANTS

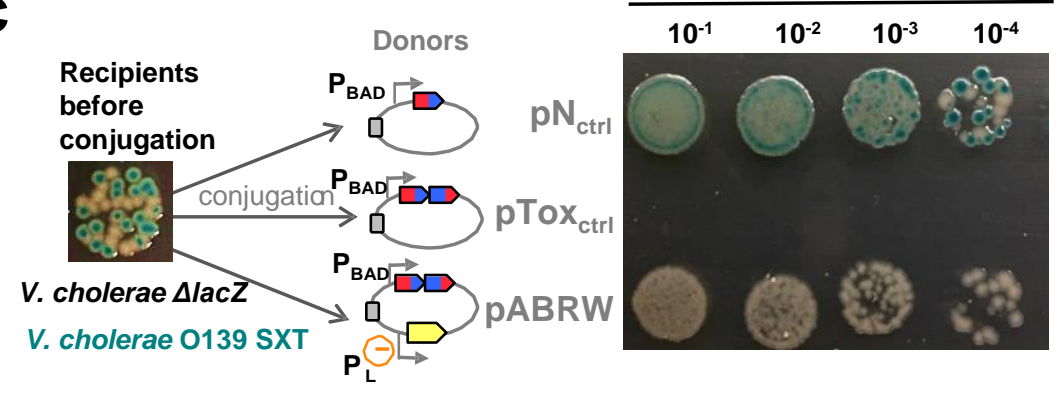

Figure 2 
a

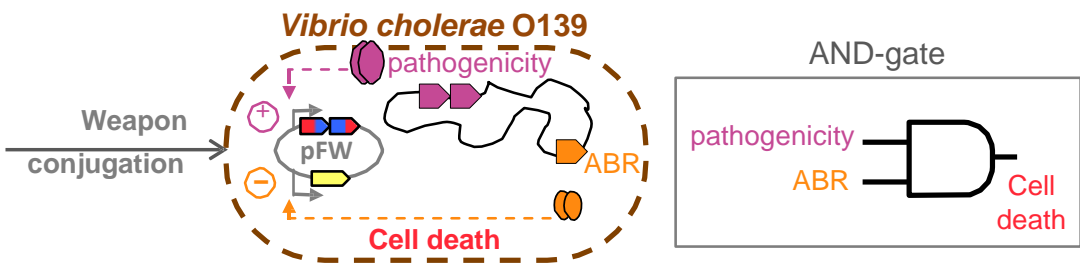

b

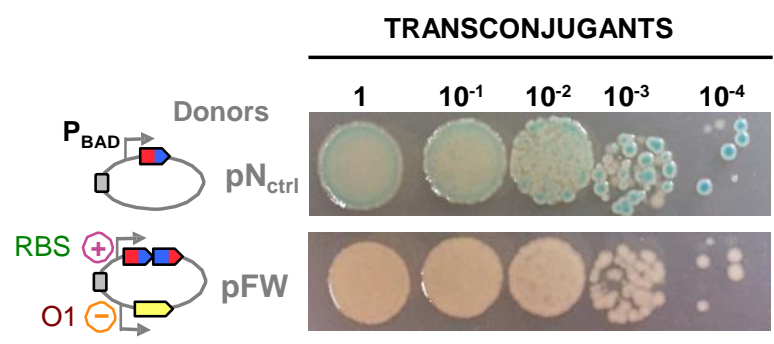

Figure 3 
a

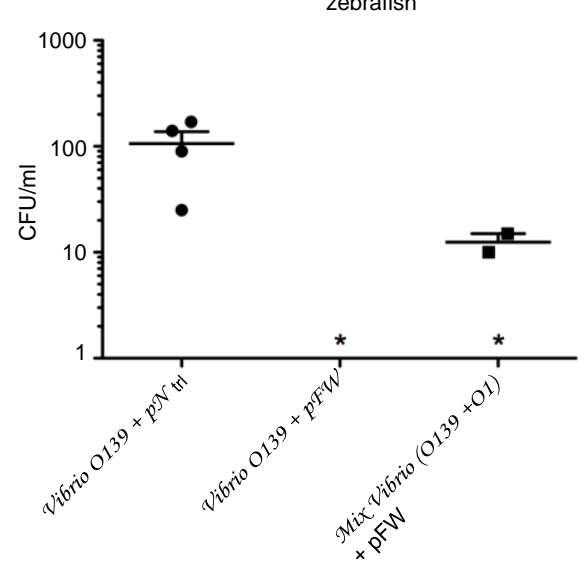

b

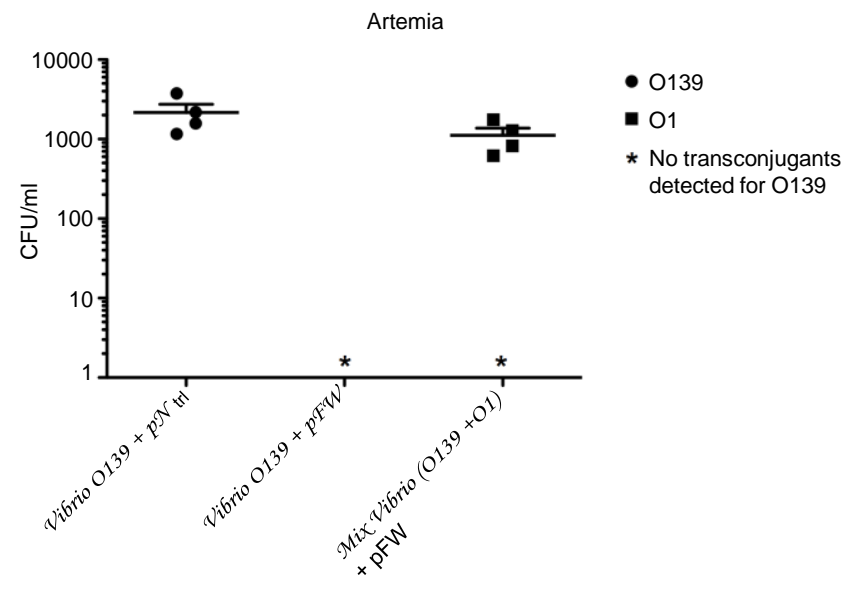

Figure 4 